\title{
The effects of occlusion and past experience on the allocation of object-based attention
}

\author{
JAY PRATT and ALLISON B. SEKULER \\ University of Toronto, Toronto, Ontario, Canada
}

\begin{abstract}
There is considerable evidence indicating that cuing a specific portion of an object results in the entire object's being attended to. In the present study, we examined whether previous experience with an object could halt perceptual (i.e., amodal) completion. In Experiment 1, two parallel rectangles were initially displayed, and then the middle portions of these objects were occluded. Attentional cuing effects were found for both discrete portions of the completed rectangles. In the final two experiments, four discrete objects were initially displayed, followed by the same occluder as that used in the first experiment. The appearance of the occluder (500 msec before the cue in Experiment 2, $100 \mathrm{msec}$ before the cue in Experiment 3) allowed the four discrete objects to be completed into two rectangles. Attentional cuing effects were found for the completed rectangles in both experiments, indicating that previous experience was not sufficient to halt the amodal completion of objects.
\end{abstract}

It is well established that target detection time can be reduced at a cued location in space, when compared with other, uncued locations (e.g., Posner, 1980; Yantis \& Jonides, 1984). Such an attentional cuing effect presumably occurs because stimulus information is extracted more rapidly and accurately at the cued location. In recent years, it has become increasingly apparent that cuing provides benefits not just for particular locations in space, but for common regions within an object as well. For example, Egly, Driver, and Rafal (1994; see also Egly, Rafal, Driver, \& Starrveveld, 1994) presented participants with two rectangles, the end of one of which was cued. As one would expect from previous research on location cuing, the largest attentional cuing effect occurred within the same object at the same location as the cue. However, when a target appeared at a different location within the cued object, a larger attentional cuing effect was seen than when a target appeared at an equally distant location in the uncued object. Thus, attentional cuing effects appear to be both space- and object-based.

The potential importance of the object-based form of attention leads one to ask: What defines an object? The answer to this question is complicated by the fact that the sensory information specifying objects is often optically incomplete. Objects frequently occlude parts of themselves and parts of neighboring objects. Despite this

This research was supported by NSERC grants to J.P. and A.B.S. We thank James Hoffman and two anonymous reviewers for helpful comments on an earlier draft of this manuscript. Correspondence regarding this manuscript should be addressed to J. Pratt, Department of Psychology, 100 St. George Street, University of Toronto, Toronto, ON, M5S 3G3 Canada (e-mail: pratt@ psych.utoronto.ca). A. B. Sekuler is now at the Department of Psychology, McMaster University, Hamilton, ON, Canada (e-mail: sekuler@mcmaster.ca). complication, people seem to have no difficulty filling in the missing information so that complete objects are perceived (Bruno, Bertamini, \& Domini, 1997; Gold, Murray, Bennett, \& Sekuler, 2000; Nakayama, Shimojo, \& Silverman, 1989; Ringach \& Shapley, 1996; Sekuler, 1994; Sekuler \& Palmer, 1992; Sekuler, Palmer, \& Flynn, 1994; Shimojo, Silverman, \& Nakayama, 1989; van Lier, 1999). If, as some researchers have suggested, visual completion occurs preattentively (Davis \& Driver, 1997; He \& Nakayama, 1992; Mattingley, Davis, \& Driver, 1997; Rensink \& Enns, 1998), one would expect the attentional cuing effect to act within discrete regions of partly occluded objects. Moore, Yantis, and Vaughan (1998) recently reported precisely such an effect. However, in the displays used by Moore et al., occluding regions were presented at a different retinal disparity from occluded regions. Consequently, depth relations were signaled not only by the pictorial cues associated with occlusion(T-junctions), but also by stereoscopic cues. Such a stimulus configuration may be important, because previous researchers have found that completion is strengthened and/or speeded by the presence of three-dimensional occluders (Bruno et al., 1997; Rensink \& Enns, 1998). Such a result may not be surprising in the context of recent physiologicalevidence. Sugita (1999) suggests that when pictorial occlusion cues are reinforced by stereo cues, neurons in the primary visual cortex (V1) respond to occluded objects as though they were complete; these same neurons do not respond in the absence of stereo cues.

Of course, in the natural world, occlusion provides relative depth information over a much larger spatial range than that for which stereopsis is effective. Thus, to understand the role of occlusion and completion in the perception of objects, it is important to understand the effect of pictorial occlusion cues with and without correspond- 
ing stereo cues. Recent studies by Behrmann, Zemel, and Mozer (1998) and Brawn and Snowden (2000) suggest that the result should hold over the two-dimensional case, as well as over the three-dimensional case. However, there are vast stimulus and task differences among these studies. For the purposes of the present study, it is important to ensure that object-based attentional cuing effects can indeed be found for a two-dimensional version of the Moore et al. task. These cuing effects were examined in the first experiment.

A second goal of the present study was to determine whether previous experience with an object could halt the completion of attentionally relevant perceptual objects. It is well established that previous experience with objects can influence perception. In his classic analysis of the perceptual laws of organization, Wertheimer $(1923 / 1950)$ discussed the role of previous experience as a grouping factor (along with the more commonly acknowledged grouping factors, such as similarity, common fate, and proximity). For example, in his description of the "factor of objective set," Wertheimer pointed out that a grouping percept may differ, depending on what the observer has just seen (similar to the modern notions of hysteresis and/or priming). Such a role for past experience in the perception of partly occluded objects has recently been suggested by Behrmann et al. (1998). They show that a previously proposed adaptive computational network model, called MAGIC, can appropriately group spatially distinct regions of partly occluded objects. Because the grouping principles MAGIC develops emerge from experience with different feature and object combinations in images during the course of learning, Behrmann et al. suggested that prior exposure could modify these grouping principles.

Some evidence in favor of this suggestion was recently put forward by Joseph and Nakayama (1999). They found that experience with an object modified the amodal representation of that object. However, the results from Joseph and Nakayama's experiments concern the amodal continuation of surfaces behind occluders, rather than amodal completion (the formation of a perceptual object), and Rensink and Enns (1998) have suggested that continuation and completion are treated differently by the visual attention system. Thus, it remains an empirical question whether amodal completion is automatic or might be influenced by recent experience with objects. This issue was addressed in Experiments 2 and 3.

\section{EXPERIMENT 1}

The purpose of the present experiment was to determine whether the object-based attentional cuing effect found previously for occluded objects could be replicated with our apparatus and procedures. Specifically, this experiment attempted an extension of Moore et al. (1998), using stimuli in which occlusion was defined only by the pictorial cue of interposition, in the absence of consistent stereoscopic depth cues.

\section{Method}

Participants. Eight University of Toronto undergraduates participated in a single 60-min session. All had normal or correctedto-normal vision, were naive as to the purposes of the experiment, and received course credit for their participation.

Apparatus and Procedure. The experiment was conducted on a 486 PC with a VGA monitor, and the participants were positioned in a chin-/headrest $44 \mathrm{~cm}$ directly in front of the monitor. The left portion of Figure 1 illustrates the sequence of events in a trial and provides schematics of the stimuli. At the beginning of each trial, a central fixation point (white, $30.5 \mathrm{~cd} / \mathrm{m}^{2}$ ) and the outlines of two rectangles (blue, $13.0 \mathrm{~cd} / \mathrm{m}^{2}$, each subtending $1.5^{\circ} \times 9^{\circ}$ ) appeared on a black $\left(0 \mathrm{~cd} / \mathrm{m}^{2}\right)$ background. The rectangles were aligned either horizontally or vertically, and the far edge of each rectangle was $4.5^{\circ}$ from the fixation point. In the completion condition, after $1,000 \mathrm{msec}$, the outline of a plus sign (white, $30.5 \mathrm{~cd} / \mathrm{m}^{2}$, with the vertical and horizontal arms subtending $1^{\circ} \times 13^{\circ}$ ) was presented so that it occluded a portion of the surface of each rectangle. Five hundred milliseconds after the onset of the occluder, a cue was presented by changing the outline of one end $\left(1.5^{\circ} \times 1.5^{\circ}\right)$ of one of the rectangles from blue to white $\left(77.0 \mathrm{~cd} / \mathrm{m}^{2}\right)$ for $100 \mathrm{msec}$. In the $n o$ completion condition, the cue was presented 1,000 msec after the onset of the initial display, and the occluding plus sign was never presented. Following a $200-\mathrm{msec}$ delay after the offset of the cue, one target and three distractors were presented at both ends of both rectangles. The target was the outline either of a large circle or a square (white, $77.0 \mathrm{~cd} / \mathrm{m}^{2}, 0.9^{\circ}$ diameter), and the distractors were the outlines of small circles and squares (white, $77.0 \mathrm{~cd} / \mathrm{m}^{2}, 0.5^{\circ} \mathrm{di}$ ameter). The participants were instructed to press the " $\mathrm{z}$ " key with the left hand if the target was the large circle and the "/" key with the right hand if the target was the large square (all responses were made on a standard QWERTY keyboard). Incorrect responses, responses less than $100 \mathrm{msec}$, and responses greater than 1,200 msec were considered errors, and a short error tone was presented if any of these occurred. The next trial began $1,000 \mathrm{msec}$ after each response.

Design. The experiment consisted of 256 trials in each of the completion and no-completion conditions. The conditions were blocked, and the order of the conditions was counterbalanced across subjects. For half of the trials in each condition the rectangles were aligned horizontally, and for the other half they were aligned vertically. The cue was equally likely to occur in any of the four ends of the rectangles. The target was presented at the cued end on $75 \%$ of the trials (valid trial), at the uncued end of the cued rectangle on $12.5 \%$ (invalid same-object trial), and at the uncued end of the uncued rectangle directly across from the cue on $12.5 \%$ (invalid differentobject trial). The target never occurred at the uncued end of the uncued rectangle that was diagonally across from the cue. The participants were explicitly informed of the relationship between the cues and the targets. The cue was equally likely to occur in any of the four possible locations, and the target was equally likely to be a circle or a square. The distractor stimuli were randomly determined on each trial. Short breaks were given after every 84 trials.

\section{Results}

The mean response times (RTs) from correct trials were analyzed with a 2 (condition, occluder or no occluder) $\times 3$ (location, valid, invalid same-object, invalid different-object) analysis of variance (ANOVA). The mean RTs appear in the top panel of Figure 2. A main effect of location was found $[F(2,14)=18.3, p<.0001]$, with the fastest RTs on valid trials and the slowest on invalid different-object trials. There was no main effect for condition $[F(1,7)<2.8, p>.14]$ and no condition $\times$ location interaction $[F(2,14)<1]$. Planned comparison 


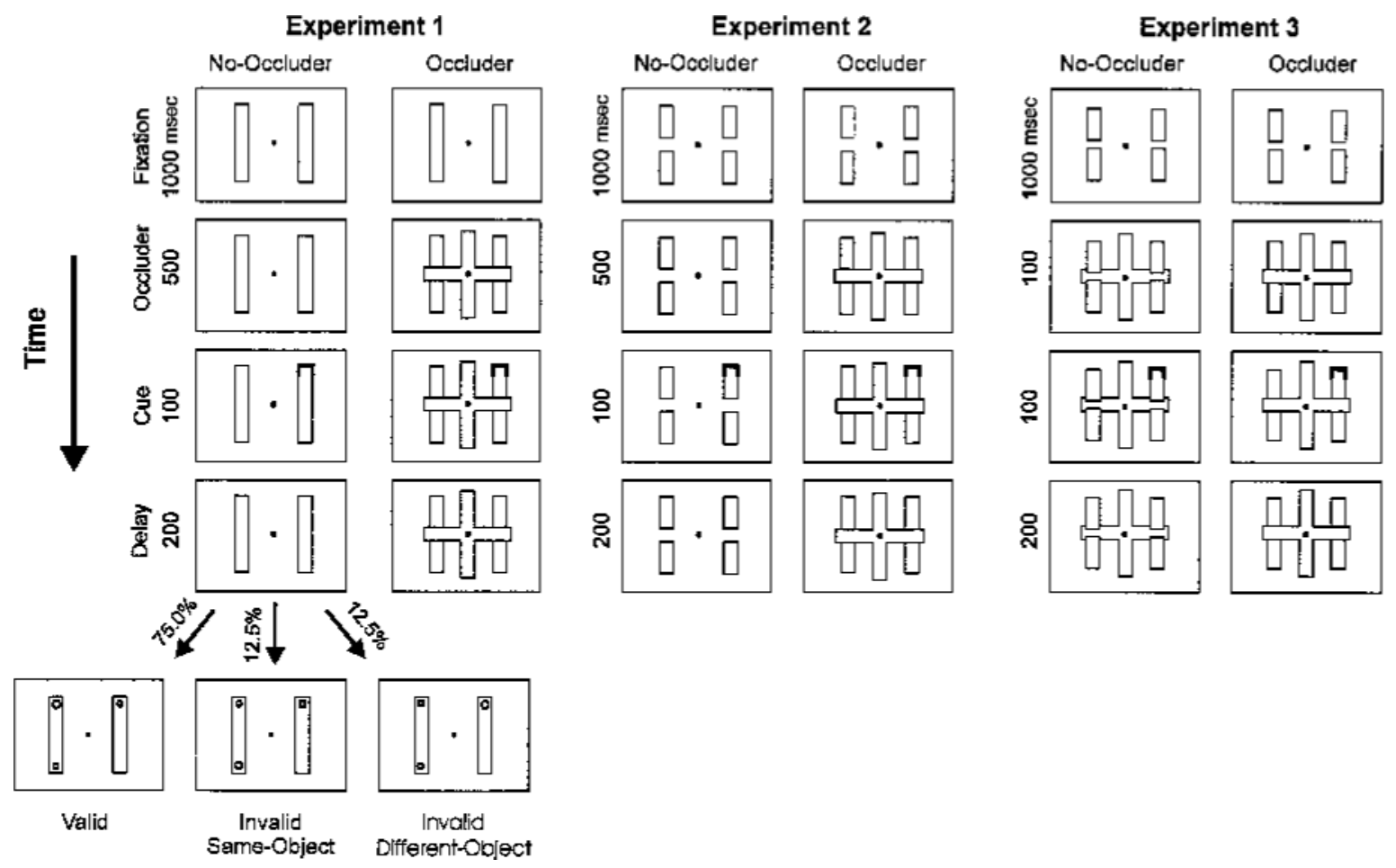

Figure 1. Trial sequences used in Experiments 1 (left portion), 2 (middle portion), and 3 (right portion). The target display is shown only for the no-occluder condition of Experiment 1 . The same target location probabilities $(75 \%$ valid, $12.5 \%$ invalid same-object, $12.5 \%$ invalid different-object), with the appropriate displays, occurred for both no-occluder and occluder conditions in Experiments 1, 2, and 3. See the text for details.

$t$ tests indicated that responses were faster for valid trials than for invalid same-object trials $(p<.002, p<$ .0015 , for occluder and no-occluder conditions, respectively), and responses were faster for invalid same-object trials than for invalid different-object trials $(p<.035$, $p<.007$, for occluder and no-occluder conditions, respectively). In addition, order effects were examined for the occluder condition. A planned comparison indicated that there was no difference in the size of the objectbased effect between occluder first-block ( $26 \mathrm{msec})$ and occluder second-block (34 msec) participants $(p>.55)$.

The percentages of errors are shown in the top portion of Table 1 and were also analyzed with a 2 (condition) $\times$ 3 (location) ANOVA. No main effects for condition

Table 1

Error Rates (Percentages) for Experiments 1, 2, and 3

\begin{tabular}{lccc}
\hline Condition & Valid & $\begin{array}{c}\text { Invalid } \\
\text { Same-Object }\end{array}$ & $\begin{array}{c}\text { Invalid } \\
\text { Different-Object }\end{array}$ \\
\hline Experiment 1 & & & \\
$\quad$ Completion & 5.75 & 4.25 & 6.13 \\
$\quad$ No completion & 5.50 & 3.63 & 5.63 \\
Experiment 2 & & & \\
$\quad$ Completion & 5.13 & 4.13 & 4.63 \\
$\quad$ No completion & 4.37 & 3.13 & 3.75 \\
Experiment 3 & & & \\
$\quad$ Completion & 4.37 & 6.47 & 3.75 \\
$\quad$ No completion & 5.50 & 3.00 & 2.25 \\
\hline
\end{tabular}

$[F(1,7)<1]$ or location $[F(2,14)<2.2, p>.15]$ were found. The interaction of condition and location also was not significant $[F(2,14)<1]$.

\section{Discussion}

The present results indicate that occluded objects received the same object-based attentional benefit as did complete objects, even when occlusion was signaled solely by pictorial cues. The results of this experiment suggest that the visual system fills in missing information behind occluders to form objects even in the absence of consistent stereo cues. Such a result is consistent with previous examinations of visual completion of objects, in which occlusion was indicated solely by pictorial cues (e.g., Behrmann et al., 1998; Brawn \& Snowden, 2000; Gold et al., 2000; Ringach \& Shapley, 1996; Sekuler, 1994; Sekuler \& Palmer, 1992; Sekuler et al., 1994; Shore \& Enns, 1997; van Lier, 1999).

\section{EXPERIMENT 2}

Experiment 2 was designed to determine whether the amodal completion of objects, as indicated by the presence of object-based attentional effects, is modified by prior experience. Unlike the two long rectangles initially presented in Experiment 1, the initial display in the present experiment consisted of four smaller rectangles. In 

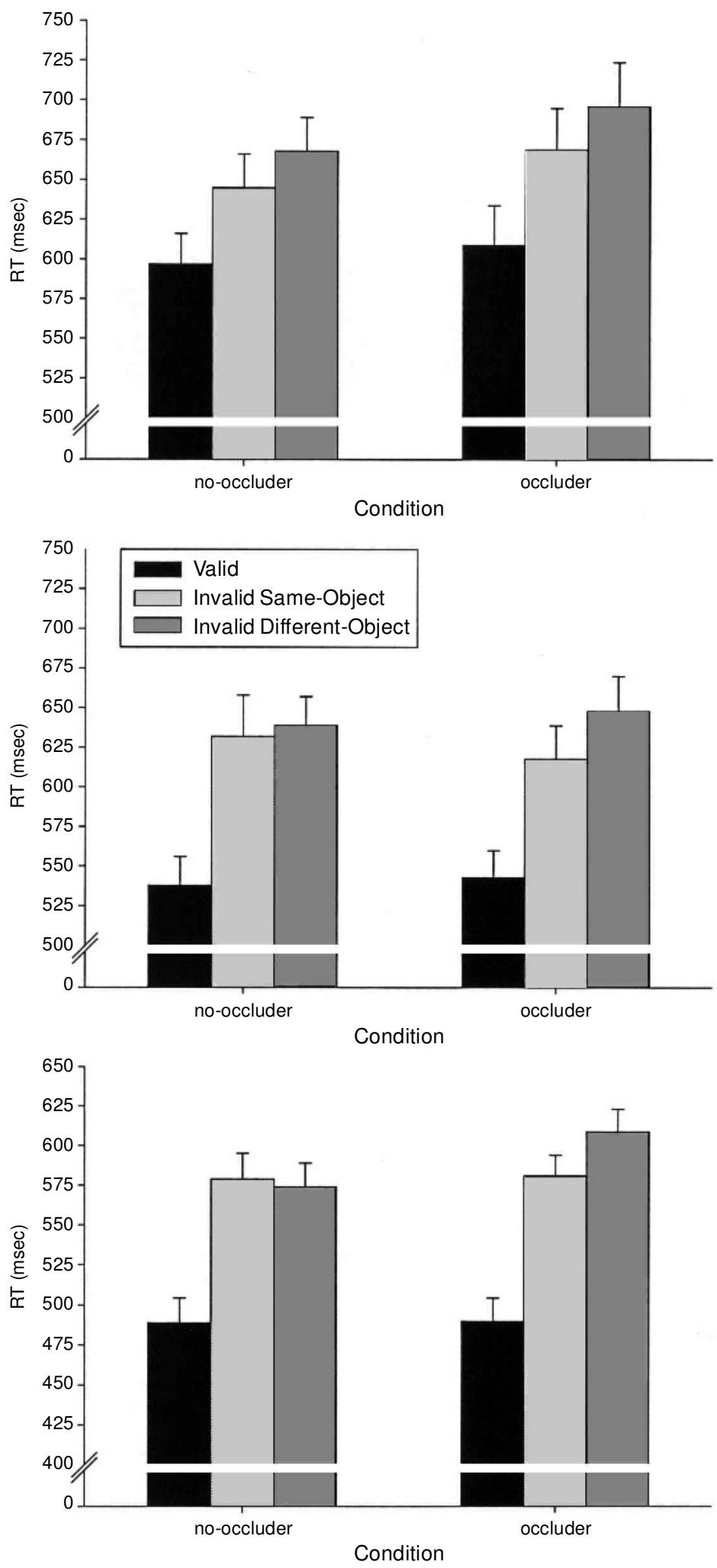

Figure 2. Mean response times (RTs) for the valid, invalid same-object, and invalid different-object conditions from Experiments 1 (top panel), 2 (middle panel), and 3 (bottom panel). Error bars indicate standard errors. 
the presence of the occluder, however, the visual display was exactly the same as the completion condition from the previous experiment (i.e., it looked as if two long rectangles were underneath an occluding cross). The question is the following: Will the attentional system treat these stimuli as two occluded objects (indicated by the visual display) or as four separate objects with an intervening occluder (indicated by prior information)? More specifically, if the attentional system treats the stimuli as two completed objects behind an occluder, the objectbased effects from the previous experiment should be found again (i.e., faster invalid same-object responses than invalid different-object responses). If the attentional system treats the stimuli as four separate objects, despite the presence of the occluder, no object-based effects should be found (i.e., equivalent RTs between invalid same-object trials and invalid different-object trials).

\section{Method}

Participants. Eight University of Toronto undergraduates participated in a single 60-min session. All had normal or correctedto-normal vision, were naive as to the purpose of the experiment, and did not participate in Experiment 1; they received course credit for their participation.

Apparatus and Procedure. The same apparatus as that in Experiment 1 was used in Experiment 2. The procedure was also the same, except that the two long rectangles used previously were replaced with four shorter rectangles (see the right portion of Figure 1). The four rectangles were $1.5^{\circ} \times 4^{\circ}$, so that the plus sign in the occluding condition occluded the space in between the pairs of rectangles up to and including the contour of their facing edges.

Design. The design of Experiment 2 was identical to that of Experiment 1 .

\section{Results}

The mean RTs from the correct trials were analyzed with a 2 (condition) $\times 3$ (location) ANOVA, and these RTs appear in the middle panel of Figure 2. A main effect of location was found $[F(2,14)=95.9, p<.0001]$, with the fastest RTs on valid trials and the slowest on invalid different-object trials. There was no main effect for condition $[F(1,7)<1]$ and no condition $\times$ location interaction $[F(2,14)<1.2, p>.34]$. Planned comparison $t$ tests indicated that, for the completion condition, responses were faster for valid trials than for invalid sameobject trials $(p<.0001)$ and responses were faster for invalid same-object trials than for invalid different-object trials $(p<.02)$. Although in the no-completion condition we found the same advantage for valid trials over invalid same-object trials $(p<.0001)$, no difference was found between invalid same-object and invalid differentobject trials $(p>.58)$. Also, a planned comparison indicated that there was no difference in the size of the objectbased effect between occluder first-block $(26 \mathrm{msec})$ and occluder second-block (34 msec) participants $(p>.65)$.

The percentages of errors are shown in the bottom portion of Table 1 and were analyzed with a 2 (condition) $\times 3$ (location) ANOVA. No main effects for condition $[F(1,7)<1]$, or location $[F(2,14)<1]$ were found.
The interaction of condition and location was also not significant $[F(2,14)<1]$.

\section{Discussion}

The results from the no-completion condition confirm that the attentional effect seen in Experiment 1 and in previous research (e.g., Egly, Driver, \& Rafal, 1994; Egly, Rafal, et al., 1994) is truly object based. When stimuli with the same spatial configuration are presented but comprise separate objects, the same advantage is no longer seen for invalid same-object targets over invalid different-object targets. If previous experience with the multiple-object configuration affected the deployment of attention, we would expect similar results in the completion condition. However, the results from the completion condition follow the same pattern of results as that seen in Experiment 1-that is, faster responses for invalid same-object targets than for invalid different-object targets. This pattern of results indicates that visual completion occurred despite preexposure to the multiple-object configuration. Such a result suggests that amodal completion is automatic; it is not influenced by recent experience with objects.

One might argue that the lack of an experience effect in our experiment was due to our choice of temporal parameters. Previous research (Murray, Sekuler, \& Bennett, 1999; Ringach \& Shapley, 1996; Sekuler \& Palmer, 1992) has shown that visual completion requires some short, but measurable, amount of time. Estimates of the required time to completion range from 60 to $200 \mathrm{msec}$, in conditions in which a time course is observed. In the present experiments, the occluder was present for $500 \mathrm{msec}$ before the cue appeared. We specifically chose this duration because we wanted to be sure the observer viewed the occluded stimulus for a long enough period to enable completion. However, the possibility remains that an experience effect would be seen at much shorter initial occlusion durations. This possibility was directly tested in Experiment 3 .

\section{EXPERIMENT 3}

The second experiment showed that prior experience was not sufficient to stop amodal completion. However, the occluder was presented for some time $(500 \mathrm{msec})$ before the cue, and it is possible that the representation of the four separate objects deteriorated in that time. To examine this possibility, the occluder in the present experiment was presented for only $100 \mathrm{msec}$ before the cue. This time interval was chosen because it should be near the minimum duration needed for completion (Murray et al., 1999; Ringach \& Shapley, 1996; Sekuler \& Palmer, 1992). In addition, the occluder object (the plus sign) was always present in both the occluder and the no-occluder conditions. In the occluder condition, the target objects appeared to lie behind the plus sign, whereas the target objects in the no-occluder condition appeared to lie in 
front of the plus sign (see Figure 1). By doing this, the amount of visual information presented in both conditions was made more comparable.

\section{Method}

Participants. Eight University of Toronto undergraduates participated in a single 60-min session. All had normal or correctedto-normal vision, were naive as to the purpose of the experiment, and did not participate in either Experiment 1 or 2; they received course credit for their participation.

Apparatus and Procedure. The same apparatus as that used previously was used in Experiment 3. The procedure was the same as that in Experiment 2, except for two alterations. The first was that the occluder was presented for $100 \mathrm{msec}$ before the cue. The second was that the occluder appeared in both the completion and the no-completion conditions, with the occluder appearing on top of the rectangles in the completion condition and below the rectangles in the no-completion condition. This way, the displays in the two conditions were more similar to each other, although amodal completion was possible only in the completion condition.

Design. The design of Experiment 2 was identical to that of Experiment 1 .

\section{Results}

The mean RTs from the correct trials were analyzed with a 2 (condition) $\times 3$ (location) ANOVA, and these RTs appear in the bottom panel of Figure 2. A main effect of location was found $[F(2,14)=52.1, p<.0001]$, with the fastest RTs on valid trials and the slowest on invalid different-object trials. There was no main effect for condition $[F(1,7)<1]$. The interaction was significant $[F(2,14)=4.1, p<.04]$. Planned comparison $t$ tests indicated that, for the completion condition, responses were faster for valid trials than for invalid same-object trials $(p<.001)$ and responses were faster for invalid same-object trials than for invalid different-object trials $(p<.005)$. Although in the no-completion condition we found the same advantage for valid trials over invalid same-object trials $(p<.002)$, no difference was found between invalid same-object and invalid different-object trials $(p>.65)$. Also, a planned comparison indicated that there was no difference in the size of the objectbased effect between occluder first-block $(26 \mathrm{msec})$ and occluder second-block (30 msec) participants $(p>.70)$.

The percentages of errors are shown in the bottom portion of Table 1 and were analyzed with a 2 (condition) $\times 3$ (location) ANOVA. No main effects for condition $[F(1,7)<2.7, p>.14]$ or location $[F(2,14)<2.7$, $p>.14]$ were found. The interaction of condition and location was also not significant $[F(2,14)<2.9, p>$ $.08]$.

With a better control condition and a much shorter duration between the appearance of the occluder and the onset of the cue, the results from the present experiment replicated the results from the second experiment. Taken together, these two experiments provide converging evidence that, at least under some circumstances, previous experience is not sufficient to stop object completion, as measured by object-based attentional effects.

\section{GENERAL DISCUSSION}

Our results support the view that visual completion precedes object-based attentional selection, even when the cue for completion is purely pictorial. Our results suggest further that object-based attentional selection is not necessarily modified by past experience with objects. These results place important constraints on the development of models of object recognition and visual attention.

For example, our results place constraints on the notion of object files (Kahneman, Treisman, \& Gibbs, 1992). One would assume that object files were initially formed when observers viewed our preview stimuli (two long rectangles in Experiment 1 or four smaller rectangles in Experiments 2 and 3). If, as was suggested by Joseph and Nakayama (1999), the object file tag remains when an occluder is added to the stimulus, one would have expected completion to be disrupted in our Experiments 2 and 3 , so that no object-based attentional cuing effect would be seen. However, the pattern of results in the occluder conditions in all the experiments were identical. Regardless of whether two or four object files were initially tagged, once the occluder was in place the attentional system treated the stimuli like two occluded objects.

Why did Joseph and Nakayama (1999) find such a striking effect of past experience where we found no effect over the same time frame? One important difference seems to be that the preview stimuli used by Joseph and Nakayama were intended to enhance visual completion, whereas our preview stimuli were intended to inhibit visual completion. In addition, the stimuli used by Joseph and Nakayama merely indicated that an object continued behind an occluder (because there were no relatable contours on the opposite side of the occluder), whereas our stimuli provided strong pictorial cues that an object should be completed behind an occluder (there were relatable contours on the opposite side of the occluder; Kellman \& Shipley, 1991). Thus, effects of past experience were found when preview objects enhanced the percept of continuation, but no effects of past experience were found when preview objects conflicted with cues for visual completion. Finally, it is important to remember that our experiment was addressing the role of previous experience on the allocation of object-based attention, whereas Joseph and Nakayama were investigating the role of previous experience on motion perception. Previous experience may differentially affect these different types of tasks.

Although one might take the present results as evidence that object files actually are not formed, it seems more reasonable to propose that our study simply illustrates one instance in which object files may be overwritten or replaced. It may be that object file tags survive only when they complement cues for visual completion but that, when the object file conflicts with completion 
cues, an effectively new object file is formed. Future studies might examine the limits of this effect-for example, by examining the relationship between the relative salience of object files and completion cues (e.g., by manipulating contour relatability, common surface characteristics, etc.).

The results of those further studies, combined with the present results, will place strong constraints on computational models of grouping and visual completion, such as MAGIC (Behrmann et al., 1998; Mozer, Zemel, Behrmann, \& Williams, 1992). If, as was suggested by Behrmann and colleagues, the grouping principles MAGIC derives can be altered by experience, our results point to a limit of that plasticity. Any modification of MAGIC, or related models, to enable short term plasticity in the allocation of attention to occluded objects must account both for the conditions under which plasticity is enabled (e.g., Joseph \& Nakayama, 1999) and for those in which it is not (the present study).

\section{REFERENCES}

Behrmann, M., Zemel, R., \& Mozer, M. (1998). Object-based attention and occlusion: Evidence from normal subjects and a computational model. Journal of Experimental Psychology: Human Perception \& Performance, 24, 1011-1036.

Brawn, P. T., \& SNOWden, R. J. (2000). Attention to overlapping objects: Detection and discrimination of luminance changes. Journal of Experimental Psychology: Human Perception \& Performance, 26, 342-358.

Bruno, N., Bertamini, M., \& Domini, F. (1997). Amodal completion of partly occluded surfaces: Is there evidence for a mosaic stage? Journal of Experimental Psychology: Human Perception \& Performance, 23,1412-1426.

DAVIS, G., \& DrIVER, J. (1997). Spreading of visual attention to modally versus amodally completed regions. Psychological Science, 8, 275281.

Egly, R, Driver, J., \& RAFAL, R. D. (1994). Shifting visual attention between objects and locations: Evidence from normal and parietal lesion subjects. Journal of Experimental Psychology: General, 123, 161-177.

Egly, R, Rafal, R, Driver, J., \& Starrveveld, Y. (1994). Covert orienting in the split brain reveals hemispheric specialization for objectbased attention. Psychological Science, 5, 380-383.

Gold, J., Murray, R. F., Bennett, P. J., \& Sekuler, A. B. (2000). Deriving behavioral receptive fields for visually completed contours. Current Biology, 10, 663-666.

He, Z J., \& NaKayama, K. (1992). Surfaces versus features in visual search. Nature, 359, 231-233.

JoSEPH, J. S., \& NAKAYAMA, K. (1999). Amodal representation depends on the object seen before partial occlusion. Vision Research, 39, 283292.
Kahneman, D., Treisman, A., \& Gibbs, B. J. (1992). The reviewing of object files: Object-specific integration of information. Cognitive Psychology, 24, 175-219.

Kellman, P., \& Shipley, T. F. (1991). A theory of visual interpolation in object perception. Cognitive Psychology, 23, 141-221.

Mattingley, J. B., Davis, G., \& Driver, J. (1997). Preattentive fillingin of visual surfaces in parietal extinction. Nature, 275, 671-674.

Moore, C. M., Yantis, S., \& Vaughan, B. (1998). Object-based visual selection: Evidence from perceptual completion. Psychological Science, 9, 104-110.

Mozer, M. C., Zemel, R. S., Behrmann, M., \& Williams, C. K. I. (1992). Learning to segment images using dynamic feature binding. Neural Computation, 4, 650-665.

Murray, R. F., Sekuler, A. B., \& Bennett, P. J. (1999). Grouping of partially occluded contours for symmetry judgement [Abstract]. Investigative Ophthalmology \& Visual Science, 40, S359.

Nakayama, K., Shimojo, S., \& Silverman, G. H. (1989). Stereoscopic depth: Its relation to image segmentation, grouping, and the recognition of occluded objects. Perception, 18, 55-68.

Posner, M. I. (1980). Orienting of attention. Quarterly Journal of Experimental Psychology, 32, 3-25.

RAMACHANDRAN, V. S., \& ANSTIS, S. (1983). Perceptual organization of moving patterns. Nature, 304, 529-531.

RENSINK, R. A., \& ENNS, J. T. (1998). Early completion of occluded objects. Vision Research, 38, 2489-2505.

Ringach, D. L., \& Shapley, R. (1996). Spatial and temporal properties of illusory contours and amodal boundary completion. Vision Research, 36, 3037-3050.

SEKULER, A. B. (1994). Local and global minima in visual completion: Effects of symmetry and orientation. Perception, 23, 529-545.

Sekuler, A. B., \& Palmer, S. E. (1992). Perception of partly occluded objects: A microgenetic analysis. Journal of Experimental Psychology: General, 121, 95-111.

Sekuler, A. B., Palmer, S. E., \& Flynn, C. (1994). Local and global processes in visual completion. Psychological Science, 5, 260-267.

Shimojo, S., Silverman, G. H., \& Nakayama, K. (1989). Occlusion and the solution to the aperture problem. Vision Research, 29, 619626.

Shore, D. I., \& ENNS, J. T. (1997). Shape completion time depends on the size of the occluded region. Journal of Experimental Psychology: Human Perception \& Performance, 23, 980-998.

SugITA, Y. (1999). Grouping of image fragments in primary visual cortex. Nature, 401, 269-272.

VAN LIER, R. (1999). Investigating global effects in visual occlusion: From a partly occluded square to the back of a tree-trunk. Acta Psychologica, 102, 203-220.

WERTHEIMER, M. (1950). Laws of organization in perceptual forms. In W. D. Ellis (Ed.), A source book of Gestalt psychology (pp. 71-88). New York: Humanities Press. (Original work published 1923)

YANTIS, S., \& JonidES, J. (1984). Abrupt visual onsets and selective attention: Evidence from visual search. Journal of Experimental Psychology: Human Perception \& Performance, 10, 601-621.

(Manuscript received April 11, 2000; revision accepted for publication March 27, 2001.) 